\title{
Purification of Recombinant $\alpha$-synuclein: A Comparison of Commonly Used Protocols
}

\author{
Amberley D. Stephens,* Dijana Matak-Vinkovic, Ana Fernandez-Villegas, \\ and Gabriele S. Kaminski Schierle*
}

Cite This: Biochemistry 2020, 59, 4563-4572

Read Online

ABSTRACT: The initial state of the intrinsically disordered protein $\alpha$-synuclein (aSyn), e.g., the presence of oligomers and degradation products, or the presence of contaminants and adducts can greatly influence the aggregation kinetics and toxicity of the protein. Here, we compare four commonly used protocols for the isolation of recombinant aSyn from Escherichia coli: boiling, acid precipitation, ammonium sulfate precipitation, and periplasmic lysis followed by ion exchange chromatography and gel filtration. We identified, using nondenaturing electrospray ionization mass spectrometry, that aSyn isolated by acid precipitation and periplasmic lysis was the purest and yielded the highest percentage of monomeric protein, $100 \%$ and $96.5 \%$, respectively. We then

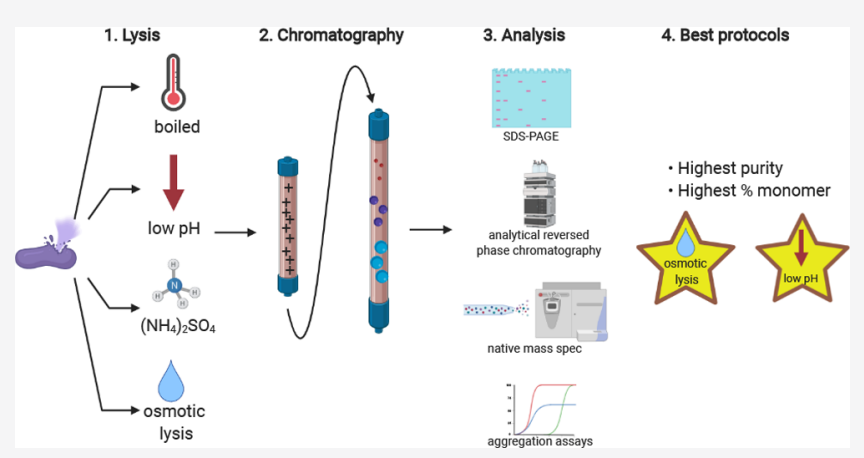
show that aSyn purified by the different protocols exerts different metabolic stresses in cells, with the more multimeric/degraded and least pure samples leading to a larger increase in cell vitality. However, the percentage of monomeric protein and the purity of the samples did not correlate with aSyn aggregation propensity. This study highlights the importance of characterizing monomeric aSyn after purification, as the choice of purification method can significantly influence the outcome of a subsequent study.

$\mathrm{T}$ he protein $\alpha$-synuclein (aSyn) is the predominant protein found in insoluble aggregates called Lewy bodies and Lewy neurites in the neurons of patients suffering from synucleinopathies, such as Parkinson's disease. Major emphasis has been placed on studying how this usually soluble and intrinsically disordered protein (IDP) misfolds into highly structured $\beta$ sheet-containing fibrils. Many studies have used purified recombinant aSyn to investigate its misfolding and cellular interactions in both in vitro and in vivo experiments. However, there are currently several different recombinant aSyn purification protocols in use in the literature. Often the purified recombinant protein lacks validation and quality control prior to its use in follow-up assays, the impact of which is currently unknown.

As an IDP, it is possible to separate aSyn from other proteins using methods to precipitate structured proteins. When a sample is boiled, many proteins undergo heat denaturation where internal bonds become broken, disrupting the structure and leading to precipitation, yet heating leaves IDPs in solution due to their lack of structure. Acid shock works in a similar way by disrupting intramolecular bonds, but by altering the charge of the amino acids, which leads to altered bonding within the proteins and subsequent precipitation. Ammonium sulfate $\left[\left(\mathrm{NH}_{4}\right)_{2} \mathrm{SO}_{4}\right]$ precipitation is also used to purify aSyn. Increasing the percentage of $\left(\mathrm{NH}_{4}\right)_{2} \mathrm{SO}_{4}$ in solution leads to precipitation of different proteins at different concentrations of $\left(\mathrm{NH}_{4}\right)_{2} \mathrm{SO}_{4}$ due to favoring hydrophobic interactions and self- association. ${ }^{1}$ Periplasmic lysis of aSyn from Escherichia coli is a more recent protocol that is less "harsh" on the protein compared to heating or acid shock. aSyn is naturally trafficked to the periplasm when expressed in E. coli. To release aSyn, but not the whole cell content, the periplasm is lysed by osmotic stress. ${ }^{2}$

aSyn resides as a dynamic ensemble of conformations in its soluble form and is therefore very sensitive to its surrounding environment. $^{3,4}$ It is currently unclear whether the methods employed to purify aSyn can influence the conformations formed within the dynamic ensemble or whether they can affect which aggregation prone or non-aggregation prone pathways the aSyn monomers will take. A few studies have investigated the effect of some purification protocols on the final recombinant aSyn protein. Two studies investigating the effect of heating on the structure of aSyn showed that heating the protein to 95-100 ${ }^{\circ} \mathrm{C}$ leads to C-terminally truncated species of aSyn. ${ }^{5,6}$ However, they reported no differences in the overall structure of full length aSyn by far-ultraviolet circular dichroism (UV-CD) and nuclear magnetic resonance (NMR) spectroscopy. ${ }^{5}$ Giehm et al. ${ }^{6}$

Received: September 1, 2020

Revised: November 18, 2020

Published: November 25, 2020 
favored purification using either acidification to precipitate unwanted proteins or periplasmic lysis that resulted in a higher purity of aSyn compared to that seen with boiling. ${ }^{7}$ However, it has been observed that acidification leads to C-terminal charge collapse and alteration of long-range interactions within the aSyn monomer, although again this was observed to be reversible. ${ }^{8,9}$

It is currently not clear whether "reversible" changes to the conformation of aSyn actually do disrupt intramolecular bonding and lead to small shifts in the dynamic ensemble of aSyn conformations, which are not detected by averaging measurements such as NMR or CD, or whether they can influence cell assays, aggregation rates, and/or fibril polymorphism. We have previously shown, using the highly sensitive technique of hydrogen-deuterium exchange mass spectrometry (HDX-MS), that the method of storage does impact the monomeric aSyn structure. Lyophilization, a commonly used storage method for aSyn, leads to a compaction of aSyn monomers in comparison to freezing, even when reconstituted in buffer. The compaction was not detected with methods such as dynamic light scattering. ${ }^{10}$ Lyophilization leads to the formation of oligomers, which are different in structure to those in the frozen aSyn sample, and to an increased variability of the aggregation kinetics as measured by a ThT-based assay. Therefore, the final aSyn protein structure after purification will be crucial in terms of interpreting future experiments involving the protein.

Here, we present a comparison of four aSyn isolation methods [boiling, acid precipitation (ppt), $\left(\mathrm{NH}_{4}\right)_{2} \mathrm{SO}_{4} \mathrm{ppt}$, and periplasmic lysis followed by ion exchange chromatography (IEX) and gel filtration (GF)] by investigating the purity, proportion of monomer, effect on cells, aggregation rate, and fibril polymorphs of aSyn formed.

\section{MATERIALS AND METHODS}

E. coli Expression of Recombinant aSyn. Plasmid pT7-7 containing human aSyn cDNA was transformed into E. coli One Shot BL21 (DE3) Star (Thermo Fisher Scientific). Cultures $(0.5 \mathrm{~L})$ of E. coli in Lysogeny Broth (LB) containing carbenicillin $(100 \mu \mathrm{g} / \mathrm{mL})$ were grown at $37^{\circ} \mathrm{C}$ while being shaken at 200 $\mathrm{rpm}$ and induced for expression of aSyn when the $\mathrm{OD}_{600}$ reached $0.6-0.8$ with $1 \mathrm{mM}$ isopropyl $\beta$-thiogalactopyranoside (IPTG). After aSyn expression for $4 \mathrm{~h}$, the cells were pelleted by centrifugation at $8000 \mathrm{~g}$ for $15 \mathrm{~min}$.

Preparation of Protein Samples for Chromatography. Preparation for Precipitation. For acid ppt and $\left(\mathrm{NH}_{4}\right)_{2} \mathrm{SO}_{4} \mathrm{ppt}$ methods, first the E. coli pellet from $500 \mathrm{~mL}$ of culture was resuspended in $50 \mathrm{~mL}$ of lysis buffer [10 mM Tris and $1 \mathrm{mM}$ EDTA ( $\mathrm{pH} 7.2$ ) with protease inhibitor tablets (cOmplete, EDTA-free protease inhibitor cocktail, Merck)] and sonicated $30 \mathrm{~s}$ on and $30 \mathrm{~s}$ off for three rounds using a XL-2020 sonicator (Heat Systems). The sonicated E. coli were centrifuged at $20000 \mathrm{~g}$ for $30 \mathrm{~min}$, and the supernatant was saved.

Acid Precipitation. The $\mathrm{pH}$ of the supernatant was reduced to $\mathrm{pH} 3.5$ using $\mathrm{HCl}$, and the mixture stirred at room temperature (RT) for $20 \mathrm{~min}$ and then centrifuged at $60000 \mathrm{~g}$ for $30 \mathrm{~min}$. The $\mathrm{pH}$ of the supernatant was then increased to $\mathrm{pH}$ 7.5 with $\mathrm{NaOH}$ and stored overnight at $4{ }^{\circ} \mathrm{C}$. ${ }^{11}$

$\left(\mathrm{NH}_{4}\right)_{2} \mathrm{SO}_{4}$ Precipitation. A $47 \%(\mathrm{w} / \mathrm{v})\left(\mathrm{NH}_{4}\right)_{2} \mathrm{SO}_{4}$ solution was added to the supernatant, ${ }^{12}$ and the mixture stirred at $\mathrm{RT}$ for $20 \mathrm{~min}$ and then centrifuged at $60000 \mathrm{~g}$ for $30 \mathrm{~min}$. The pellet of protein was resuspended in $60 \mathrm{~mL}$ of dialysis buffer $[10 \mathrm{mM}$ Tris and $1 \mathrm{mM}$ EDTA ( $\mathrm{pH} 7.5)]$ and dialyzed overnight against the same buffer at $4{ }^{\circ} \mathrm{C}$.

Boiling. For isolation of aSyn by boiling, the E. coli pelleted from a $500 \mathrm{~mL}$ culture was resuspended in $50 \mathrm{~mL}$ of high-salt buffer [0.75 M NaCl, $100 \mathrm{mM}$ MES, and $1 \mathrm{mM}$ EDTA ( $\mathrm{pH}$ $7)],{ }^{13}$ boiled in a water bath for $20 \mathrm{~min}$ at $100{ }^{\circ} \mathrm{C}$, and then centrifuged at $60000 \mathrm{~g}$ for $30 \mathrm{~min}$. The supernatant was dialyzed against $10 \mathrm{mM}$ Tris and $1 \mathrm{mM}$ EDTA $(\mathrm{pH} 7.5)$ overnight at $4{ }^{\circ} \mathrm{C}$ in SnakeSkin dialysis tubing, with a molecular weight cutoff (MWCO) of $10000 \mathrm{Da}$ (Thermo Fisher Scientific).

Periplasmic Lysis. aSyn was released from the E. coli periplasm by osmotic lysis. ${ }^{2}$ The pellet of E. coli from a 500 $\mathrm{mL}$ culture that had not been frozen was resuspended in $100 \mathrm{~mL}$ of osmotic shock buffer [ $30 \mathrm{mM}$ Tris, $40 \%$ sucrose (w/v), and 2 $\mathrm{mM}$ EDTA ( $\mathrm{pH} \mathrm{7.2)]} \mathrm{and} \mathrm{incubated} \mathrm{at} \mathrm{RT} \mathrm{for} 10 \mathrm{~min}$. The solution was centrifuged at $18000 \mathrm{~g}$ for $20 \mathrm{~min}$. The supernatant was discarded, and the pellet resuspended in $90 \mathrm{~mL}$ of ice-cold $\mathrm{dH}_{2} \mathrm{O}$ with $37.5 \mu \mathrm{L}$ of saturated $\mathrm{MgCl}_{2}$ and kept on ice for $3 \mathrm{~min}$ before being centrifuged at $18000 \mathrm{~g}$ for $20 \mathrm{~min}$. The supernatant was dialyzed overnight against $10 \mathrm{mM}$ Tris and $1 \mathrm{mM}$ EDTA $(\mathrm{pH} 7.5)$ at $4{ }^{\circ} \mathrm{C}$. The use of EDTA is particularly important after the addition of $\mathrm{MgCl}_{2}$ as it influences structure and aggregation rates. ${ }^{14}$

Ion Exchange Chromatography. All buffers and aSyn samples for chromatography were filtered through a $0.22 \mu \mathrm{m}$ filter and degassed before use. For ion exchange chromatography (IEX), the protein was loaded onto a HiPrep QFF 16/10 anion exchange column (GE Healthcare, Uppsala, Sweden) and washed with IEX buffer A [10 mM Tris , ( $\mathrm{pH} 7.5)]$ to remove unbound proteins before aSyn was eluted against a linear gradient of 7 column volumes (CV) of IEX buffer B [10 mM Tris and $0.75 \mathrm{M} \mathrm{NaCl}(\mathrm{pH} 7.5)$ ] followed by $2 \mathrm{CV}$ of $100 \%$ IEX buffer B using an ÄKTA Pure fast protein liquid chromatography (FPLC) system (GE Healthcare). To determine the point of elution of aSyn from the chromatography column, protein fractions that were collected and monitored for absorption at $280 \mathrm{~nm}$ were run on a $4 \%$ to $12 \%$ Bis-Tris gel (Invitrogen, Thermo Fisher) using sodium dodecyl sulfate-polyacrylamide gel electrophoresis (SDS-PAGE) and stained with Coomassie blue. Fractions containing protein bands corresponding to the predicted monomeric aSyn molecular weight (MW) of $14.4 \mathrm{kDa}$ were further used in chromatographic steps. Fractions containing aSyn were pooled together and either dialyzed overnight in $20 \mathrm{mM}$ Tris ( $\mathrm{pH} 7.2$ ), concentrated with a $10 \mathrm{kDa}$ MWCO centrifugal concentrator to the desired concentration, $\sim 130-140 \mu \mathrm{M}$, and stored at $-80{ }^{\circ} \mathrm{C}$ or directly concentrated before gel filtration.

Hydrophobic Interaction Chromatography. For aSyn isolated from the periplasm, we further optimized the purification protocol to yield a higher purity of aSyn by the addition of a hydrophobic interaction chromatography (HIC) step. We changed the counterion from $0.75 \mathrm{M} \mathrm{NaCl}$ to $0.15 \mathrm{M}$ $\left(\mathrm{NH}_{4}\right)_{2} \mathrm{SO}_{4}$ during IEX chromatography to remove a dialysis step needed to exchange salts before subsequent HIC. The amount of $\left(\mathrm{NH}_{4}\right)_{2} \mathrm{SO}_{4}$ in the aSyn solution after IEX was calculated on the basis of the percentage of buffer $\mathrm{B}$ at which it eluted. On the basis of the volume of aSyn protein collected, the amount of $\left(\mathrm{NH}_{4}\right)_{2} \mathrm{SO}_{4}$ needed to make the solution up to $1 \mathrm{M}$ was calculated and then gradually added while the mixture was being stirred at room temperature.

For HIC, the $\mathrm{pH}$ of the aSyn sample was adjusted to 7 and the sample filtered through a $0.22 \mu \mathrm{m}$ filter before being loaded onto a HiPrep Phenyl FF 16/10 (High Sub) column (GE Healthcare) 
and eluted in HIC buffer A [50 mM Bis-Tris and $1 \mathrm{M}$ $\left.\left(\mathrm{NH}_{4}\right)_{2} \mathrm{SO}_{4}(\mathrm{pH} 7)\right]$ against a linear gradient of $7 \mathrm{CV}$ of $\mathrm{HIC}$ buffer B [50 mM Bis-Tris ( $\mathrm{pH} 7$ )] followed by 2 CV of $100 \%$ HIC buffer B. Fractions containing aSyn were pooled and extensively dialyzed against $20 \mathrm{mM}$ Tris ( $\mathrm{pH} 7.2$ ) overnight at 4 ${ }^{\circ} \mathrm{C}$. The protein solution was concentrated in $10000 \mathrm{Da}$ MWCO centrifugal concentrators to the desired concentration, $\sim 130-$ $140 \mu \mathrm{M}$, and frozen at $-80{ }^{\circ} \mathrm{C}$ until further use.

Gel Filtration. An aliquot of aSyn was defrosted, and $500 \mu \mathrm{L}$ of aSyn was injected into a gel filtration (GF) column, Superdex 75 10/300 GL (GE Healthcare). The sample was eluted by isocratic elution at a rate of $0.8 \mathrm{~mL} / \mathrm{min}$ in $20 \mathrm{mM}$ Tris ( $\mathrm{pH} 7.2)$. Tubing between the injection point and the fraction collector on the ÄKTA Pure FPLC system was changed from orange (0.5 $\mathrm{mm})$ to blue $(0.25 \mathrm{~mm})$ to reduce dilution of the protein sample and to give a narrower collection peak. Monomeric aSyn eluted at $\sim 9 \mathrm{~mL}$.

Densitometry to Determine the Purity of aSyn. Fractions of proteins samples were run on $4 \%$ to $12 \%$ Bis-Tris gels using SDS-PAGE for separation of proteins on the basis of size. The gels were stained with Coomassie blue, and the gel image was analyzed using Image ${ }^{15}$ to determine the percentage of aSyn present. Regions of interest were selected, and a histogram of the intensity of the dyed protein in the area was generated. From the histogram, the area of aSyn was calculated as a percentage of the total area of stained proteins to give the percentage purity.

Reversed Phase High-Pressure Liquid Chromatography to Determine the Purity of aSyn. The purity of the aSyn samples was analyzed by analytical reversed phase chromatography (aRP) on a model 1260 Infinity high-pressure liquid chromatography (HPLC) system (Agilent Technologies LDA UK Ltd.), equipped with an autosampler and a diode-array detector; $50 \mu \mathrm{L}$ of sample was injected onto a Discovery BIO Wide Pore C18 column $(15 \mathrm{~cm} \times 4.6 \mathrm{~mm}, 5 \mu \mathrm{m}$ column with a guard column) (Supelco, Merck) and eluted with a gradient of 95\% water and $0.1 \%$ acetic acid and $5 \%$ acetonitrile and $0.1 \%$ acetic acid to $5 \%$ water and $0.1 \%$ acetic acid and $95 \%$ acetonitrile and $0.1 \%$ acetic acid at a flow rate of $0.8 \mathrm{~mL} / \mathrm{min}$ over $40 \mathrm{~min}$. The elution profile was monitored by UV absorption at 220 and $280 \mathrm{~nm}$. The area under the peaks in the chromatograph of absorption at $280 \mathrm{~nm}$ was calculated to provide the percentage purity of aSyn. aSyn eluted at $\sim 17.9$ min.

Native Mass Spectrometry. Nondenaturing nanoelectrospray ionization mass spectrometry (native mass spectrometry) was used to analyze the oligomerization states of recombinant aSyn prepared using four different methods: boiling, $\left(\mathrm{NH}_{4}\right)_{2} \mathrm{SO}_{4} \mathrm{ppt}$, acid ppt, and periplasmic lysis. Native mass spectra were recorded on a Synapt HD mass spectrometer (Waters, Manchester, U.K.) modified for studying high masses. Protein samples were exchanged into a $0.2 \mathrm{M}$ ammonium acetate ( $\mathrm{pH}$ 7.0) solution using Micro Bio-Spin 6 chromatography columns (Bio-Rad, Hercules, CA) and diluted to a final concentration of 5-10 $\mu \mathrm{M}$ before analysis. An aliquot of $2.5 \mu \mathrm{L}$ of a protein solution was electrosprayed from a borosilicate emitter (Thermo Scientific) for sampling. Typical conditions for the data acquired were as follows: capillary voltage of 1.6-2.2 $\mathrm{kV}$, cone voltage of $160-190 \mathrm{~V}$, trap voltage of $40-50 \mathrm{~V}$, and transfer voltage of $140 \mathrm{~V}$ with a backing pressure of 3-4 mbar and a source temperature of $20{ }^{\circ} \mathrm{C}$. Spectra were calibrated externally using cesium iodide. Data acquisition and processing were performed using MassLynx 4.1. Spectra were edited manually using Adobe Illustrator for the purpose of this publication.

Cell Vitality Assay. Human neuroblastoma cells (SH-SY5Y) were obtained from the European Collection of Cell Cultures (ECACC, Sigma-Aldrich, Dorset, U.K.) and grown in a 1:1 mixture of minimal essential medium (MEM) (Sigma-Aldrich) and nutrient mixture Ham's F-12 (Sigma-Aldrich) supplemented with $15 \%$ FBS, $1 \%$ non-essential amino acids, $2 \mathrm{mM}$ GlutaMAX, and 1\% antibiotic-antimycotic (all from Thermo Fisher Scientific, Epsom, U.K.). The vitality of SH-SY5Y cells after treatment with aSyn was determined using an MTT [3(4,5-dimethylthiazol-2-yl)-2,5-diphenyltetrazolium bromide] kit from Promega (Madison, WI). Live cells with active metabolism convert MTT to formazan. Cells $\left(3 \times 10^{4}\right)$ were seeded into each well of 96-well culture plates overnight followed by incubation of aSyn for $24 \mathrm{~h}$. MTT was added to cells for $4 \mathrm{~h}$, and the absorption was measured with a microplate reader at $590 \mathrm{~nm}$ (Envision, PerkinElmer). The absorbance is proportional to the number of viable cells; therefore $>100 \%$ vitality may be due to an increase in the number of cell compared to the control as these are nondifferentiated neuroblastoma cells. Each experiment was performed three times using duplicates for aSyn samples from each purification batch.

Thioflavin-T-Based Kinetic Aggregation Assays. Twenty micromolar freshly made thioflavin-T (ThT) (abcam, Cambridge, U.K) was added to $50 \mu \mathrm{L}$ of $20 \mu \mathrm{M}$ aSyn after GF in $140 \mathrm{mM} \mathrm{KCl}$ and $20 \mathrm{mM}$ Tris (pH 7.2). All samples were loaded onto nonbinding, clear bottom, 96-well half-area plates (Greiner Bio-One $\mathrm{GmbH}$ ). The plates were sealed with a SILVERseal aluminum microplate sealer (Grenier Bio-One $\mathrm{GmbH}$ ). Fluorescence measurements were taken using a FLUOstar Omega plate reader (BMG LABTECH $\mathrm{GmbH}$, Ortenbery, Germany). The plates were incubated at $37{ }^{\circ} \mathrm{C}$ with double orbital shaking at $300 \mathrm{rpm}$ for $5 \mathrm{~min}$ before each read every hour for $170 \mathrm{~h}$. Excitation was set at $440 \mathrm{~nm}$ with 20 flashes, and the ThT fluorescence intensity was measured at $480 \mathrm{~nm}$ emission with a 1300 gain setting. ThT-based assays were repeated twice using four wells for each condition. For aSyn isolated by boiling, four gel-filtered samples were used. For aSyn isolated by $\left(\mathrm{NH}_{4}\right)_{2} \mathrm{SO}_{4} \mathrm{ppt}$, three gel-filtered samples were used. For aSyn isolated by acid ppt, two gel-filtered samples were used. For aSyn isolated by periplasmic lysis, three gel-filtered samples were used. Data were normalized to the sample with the maximum fluorescence intensity for each plate.

Analytical Size Exclusion Chromatography to Determine the Remaining aSyn Monomer Concentration after Aggregation Assays. At the end of the ThT-based aggregation assays, the amount of remaining monomer of aSyn in each well was determined by analytical size exclusion chromatography with HPLC (SEC-HPLC). The contents of each well after the ThT-based assay were centrifuged at $21000 \mathrm{~g}$ for $20 \mathrm{~min}$, and the supernatant was added to individual aliquots in the autosampler of the Agilent 1260 Infinity HPLC system (Agilent Technologies LDA UK Ltd.). Twenty-five microliters of each sample was injected onto an Advance Bio SEC column $(7.8 \mathrm{~mm} \times 300 \mathrm{~mm}, 300 \AA$, Agilent $)$ in $20 \mathrm{mM}$ Tris $(\mathrm{pH} 7.2)$ at a flow rate of $1 \mathrm{~mL} / \mathrm{min}$. Injections were also made for each sample at the start of the assay to quantify the amount of starting protein. The elution profile was monitored by UV absorption at 220 and $280 \mathrm{~nm}$. The remaining monomer percentage was calculated from the ratio of the area under the curves at the beginning and the end of the assay. 
a. i.

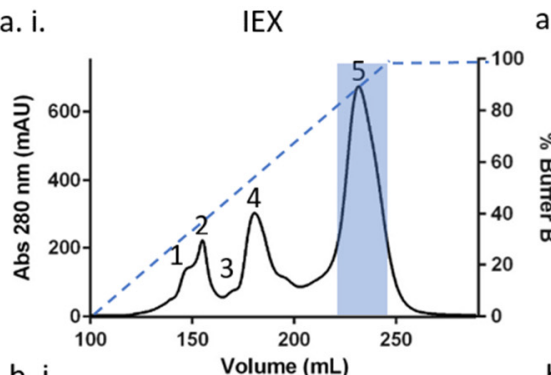

b. i.

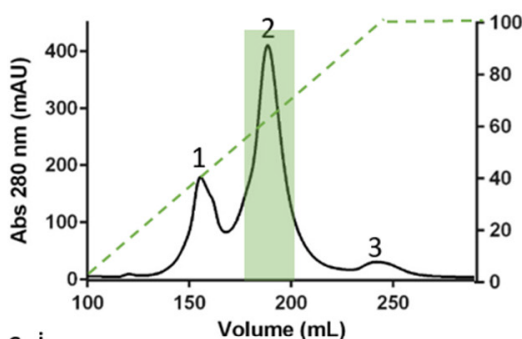

c. i.

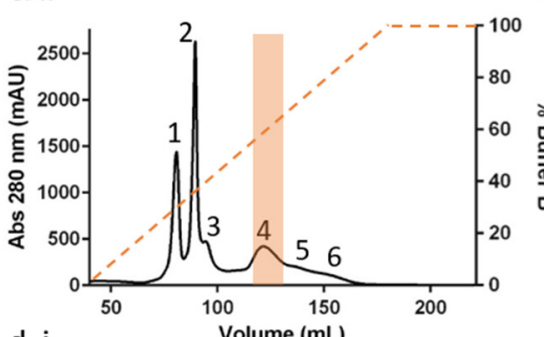

d. i.

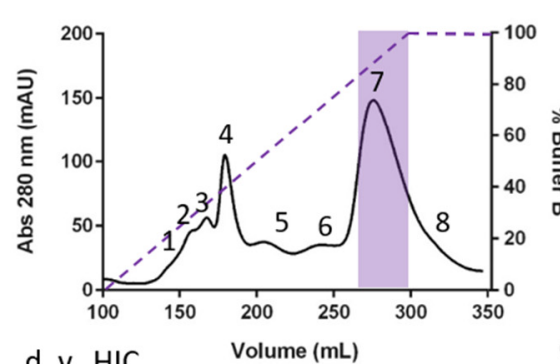

d. v. HIC

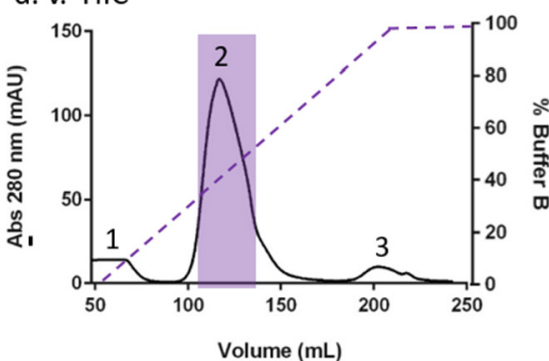

a. ii.

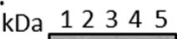

260

190

50

40

15

b. ii.

kDa 123

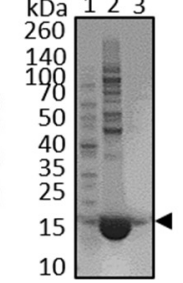

c. ii.

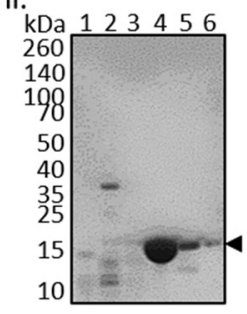

d. ii.

kDa 12345678

260

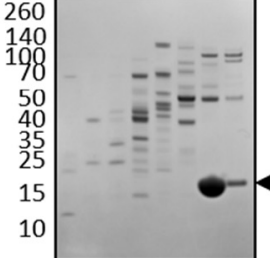

d. vi.

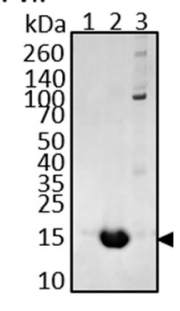

a. iii.

GF

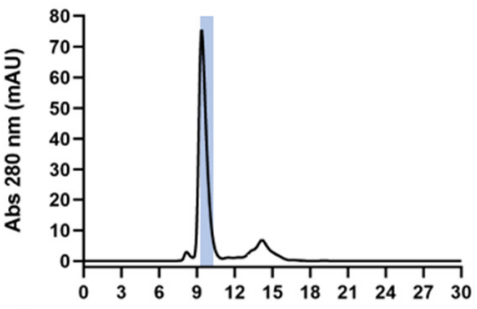

b. iii.

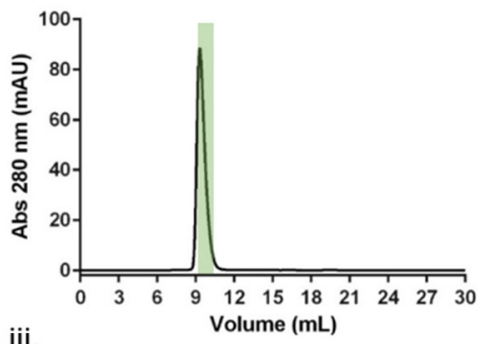

c. iii.

d. iii.
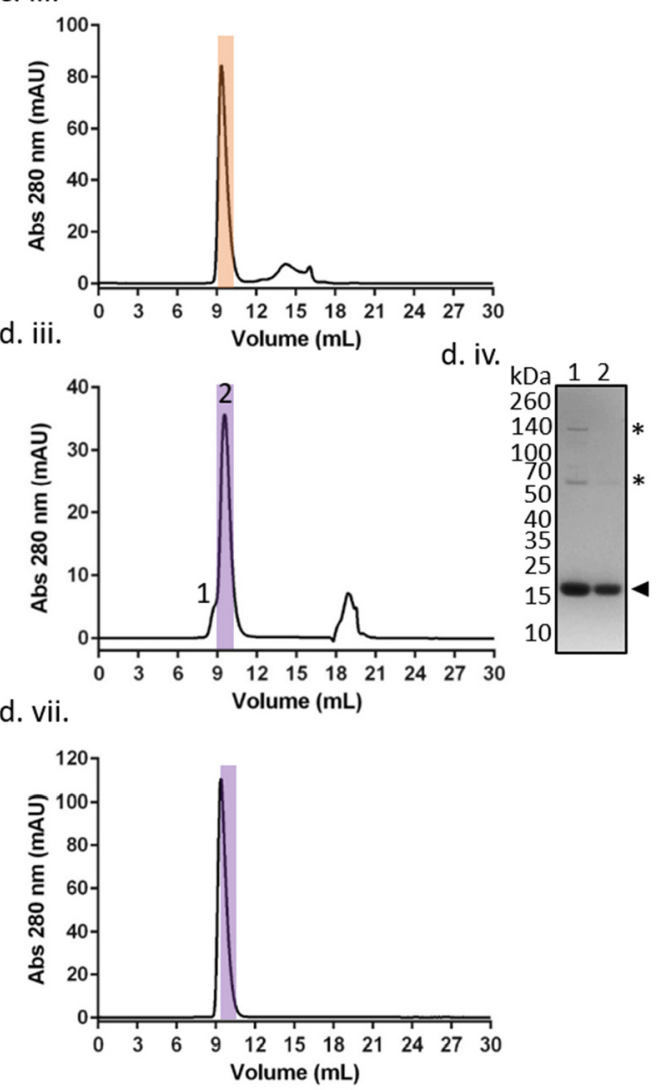

Figure 1. aSyn isolated by boiling, acid ppt, and $\left(\mathrm{NH}_{4}\right)_{2} \mathrm{SO}_{4}$ ppt is highly pure after IEX and GF, while aSyn isolated by periplasmic lysis requires an additional HIC step to increase purity. aSyn was purified by IEX chromatography from samples that were (a.i.) boiled, (b.i.) precipitated by $\left(\mathrm{NH}_{4}\right)_{2} \mathrm{SO}_{4}$, (c.i.) precipitated by acidification, and (d.i.) lysed from the periplasm. Protein fractions from IEX were taken from individual peaks with maximum absorption at $280 \mathrm{~nm}$ and analyzed by SDS-PAGE using a 4-12\% Bis-Tris gel that was stained by Coomassie blue and (a.ii.) boiled, (b.ii.) precipitated by $\left(\mathrm{NH}_{4}\right)_{2} \mathrm{SO}_{4}$, (c.ii.) precipitated by acidification, and (d.ii.) lysed from the periplasm. aSyn ran at $\sim 15 \mathrm{kDa}$, indicated by the arrow next to the gel images, and the peak in which aSyn resided is highlighted in color on the IEX chromatographs. GF of pooled fractions containing aSyn after IEX shows monomeric aSyn eluting after $\sim 9 \mathrm{~mL}$ : (a.iii.) boiled, (b.iii.) precipitated by $\left(\mathrm{NH}_{4}\right)_{2} \mathrm{SO}_{4}$, (c.iii.) precipitated by acidification, and (d.iii.) periplasmic lysis. GF of aSyn isolated by periplasmic lysis did not yield as highly pure aSyn as the other methods did, (d.iv.) as indicated by the presence of contaminating proteins (asterisks) in the Coomassie blue-stained gel. Therefore, an additional (d.v.) HIC step was added, and (d.vi.) aSyn was subsequently purer as shown by the Coomassie blue-stained gel. (d.vii.) The final GF of aSyn isolated by periplasmic lysis after IEX and HIC showed a single peak of monomeric aSyn eluting at $\sim 9 \mathrm{~mL}$.

Transmission Electron Microscopy. Twenty microliters of aSyn was taken directly from the ThT-based aggregation assay plates of the boiled, $\left(\mathrm{NH}_{4}\right)_{2} \mathrm{SO}_{4} \mathrm{ppt}$, and periplasmic lysis samples and diluted 1:4 with $\mathrm{dH}_{2} \mathrm{O}$. The acid ppt aSyn sample was used neat, and all samples were incubated on glowdischarged carbon-coated copper grids for 1 min before being 
washed twice with $\mathrm{dH}_{2} \mathrm{O}$. Then, $2 \%$ uranyl acetate was used to stain the samples for $30 \mathrm{~s}$ before they were imaged on the Tecnai G2 $80-200 \mathrm{kv}$ transmission electron microscopy (TEM) instrument at the Cambridge Advanced Imaging Centre.

\section{RESULTS}

Acid Precipitation of $E$. coli Proteins Leads to the Highest Purity of aSyn before Chromatographic Separation. Four commonly used protocols for the purification of recombinant aSyn were compared to determine which protocol yielded the highest proportion of monomeric aSyn and the highest degree of purity. First, in all four protocols, $0.5 \mathrm{~L}$ of $E$. coli culture was induced for $4 \mathrm{~h}$ with IPTG before the bacteria were pelleted. The pellets were then treated differently depending on the isolation protocol. For boiled samples, the E. coli pellet was resuspended in a high-salt buffer, boiled in a water bath at 100 ${ }^{\circ} \mathrm{C}$ for $20 \mathrm{~min}$, and centrifuged. The supernatant was dialyzed overnight in $10 \mathrm{mM}$ Tris and $1 \mathrm{mM}$ EDTA ( $\mathrm{pH} \mathrm{7.5)}$. For the acid and $\left(\mathrm{NH}_{4}\right)_{2} \mathrm{SO}_{4}$ ppt protocols, the E. coli pellets were resuspended in $10 \mathrm{mM}$ Tris and $1 \mathrm{mM}$ EDTA ( $\mathrm{pH} 7.5$ ) including protease inhibitors before being sonicated and centrifuged. The supernatant was then precipitated either by reducing the $\mathrm{pH}$ to 3.5 with $\mathrm{HCl}$ on a stirrer for $20 \mathrm{~min}$ or by adding $47 \%(\mathrm{w} / \mathrm{v})\left(\mathrm{NH}_{4}\right)_{2} \mathrm{SO}_{4}$ on a stirrer for $20 \mathrm{~min}$. The precipitates were centrifuged, and the supernatant from the acid ppt was brought back to neutral $\mathrm{pH}$ with $\mathrm{NaOH}$ and stored overnight at $4{ }^{\circ} \mathrm{C}$ before chromatography was performed. The pellet from the $\left(\mathrm{NH}_{4}\right)_{2} \mathrm{SO}_{4}$ ppt containing aSyn was resuspended in $10 \mathrm{mM}$ Tris and $1 \mathrm{mM}$ EDTA (pH 7.5) and dialyzed overnight in the same buffer. For periplasmic lysis, the E. coli periplasm was lysed by osmotic shock. The E. coli pellet was resuspended in a sucrose-based buffer that acts as an osmotic stabilizer preventing whole cell lysis. ${ }^{16}$ After centrifugation to pellet the $E$. coli culture, the outer membrane was lysed by osmotic shock with water and $\mathrm{MgCl}_{2}$ to release the contents of the periplasm, but not the cytoplasm. The lysed protein was dialyzed overnight in $10 \mathrm{mM}$ Tris and $1 \mathrm{mM}$ EDTA ( $\mathrm{pH} 7.5$ ). The acid-precipitated aSyn sample was the most pure at this point, with $96.5 \%$ purity by densitometry measurement of the SDS-PAGE Coomassie-stained gel, which agrees with a previous study ${ }^{7}$ (Figure S1 and Table S1).

Chromatographic Isolation of aSyn Yields 80-95\% Pure aSyn. IEX was then used to isolate aSyn from all protein solutions using a HiPrep QFF 16/10 anion exchange column. aSyn was eluted on a linear gradient of IEX buffer A [10 mM Tris (pH 7.5)] against IEX buffer B [10 mM Tris and $0.75 \mathrm{M} \mathrm{NaCl}$ ( $\mathrm{pH} 7.5)$ ] (Figure 1a.i-d.i). To determine the fractions in which aSyn resided, the samples were analyzed by SDS-PAGE and the gel was stained by Coomassie blue to visualize the protein. Fractions containing aSyn are highlighted in the colored block on the IEX chromatograms (Figure 1a.i,ii-d.i,ii). The purity of the samples was analyzed by densitometry, and the aSyn precipitated in acid and the aSyn that was boiled were found to be $100 \%$ and $99.3 \%$ pure, respectively (Table S1). After IEX, aSyn samples were dialyzed in $20 \mathrm{mM}$ Tris ( $\mathrm{pH} \mathrm{7.2)}$ and concentrated using centrifugal concentrators with a MWCO of $10 \mathrm{kDa}$. aSyn was concentrated until the protein concentration was between 130 and $140 \mu \mathrm{M}$ before being stored at $-80^{\circ} \mathrm{C}$. To increase the purity of the aSyn from samples isolated by periplasmic lysis and $\left(\mathrm{NH}_{4}\right)_{2} \mathrm{SO}_{4}$ ppt further, and to ensure isolation of monomeric protein, gel filtration was used. $500 \mu \mathrm{L}$ of aSyn was injected onto a Superdex 75 10/300 GL gel filtration column and eluted isocratically (Figure 1a.iii-d.iii). The
Coomassie blue-stained gel after SDS-PAGE of aSyn showed that all isolation protocols, apart from periplasmic lysis (Figure 1d.iv), led to $100 \%$ pure aSyn after IEX and GF (Figure $2 \mathrm{a}$ and

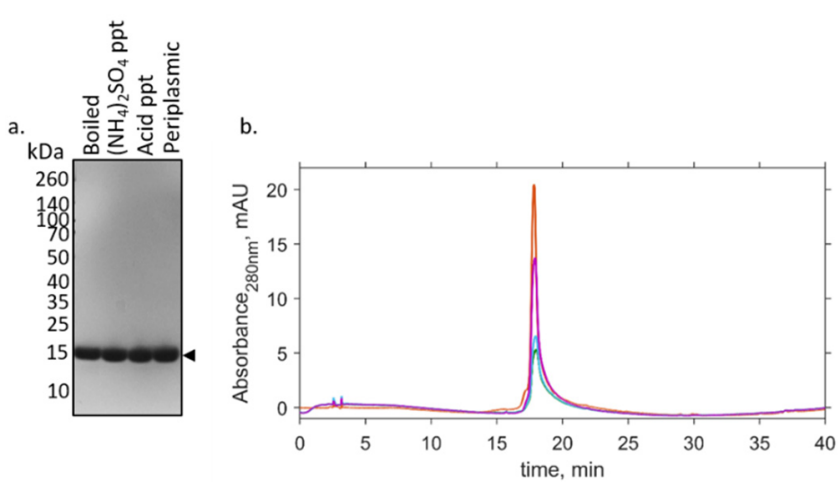

Figure 2. Coomassie blue-stained gel and reverse phase chromatographs of aSyn after gel filtration show highly pure monomeric aSyn. (a) Samples of aSyn after gel filtration were analyzed by SDS-PAGE on a $4 \%$ to $12 \%$ Bis-Tris gel and stained with Coomassie blue. aSyn appears as a single band, indicated by the arrow at $\sim 15 \mathrm{kDa}$. (b) Fifty microliters of each sample was injected onto an analytical Discovery BIO Wide Pore $\mathrm{C} 18$ column to determine the purity of aSyn by analytical reverse phase chromatography. aSyn isolated by boiling (blue) was $86 \%$ pure. aSyn isolated by $\left(\mathrm{NH}_{4}\right)_{2} \mathrm{SO}_{4}$ ppt (green) was $81 \%$ pure. aSyn isolated by acid ppt (orange) was $89.9 \%$ pure. aSyn isolated from periplasmic lysis (purple) was 95\% pure (all determined by the area under the peak).

Table S1). The aSyn sample isolated by periplasmic lysis still contained contaminating proteins; aSyn was only $91.7 \%$ pure in fraction 1 and $97.7 \%$ pure in fraction 2 (stars in Figure 1d.iv indicate the contaminants; Table S1). Previous protocols using the periplasmic lysis protocol have also employed an extra hydrophobic interaction chromatography (HIC) step. ${ }^{17}$ An additional HIC step was added using a HiPrep Phenyl Fast Flow (high sub) $16 / 10$ column, but the previous protocol was updated to save time by substituting the counterion salt in IEX from $\mathrm{NaCl}$ to $\left(\mathrm{NH}_{4}\right)_{2} \mathrm{SO}_{4}$ to prevent an additional buffer exchange step before HIC. Therefore, directly after IEX, $\left(\mathrm{NH}_{4}\right)_{2} \mathrm{SO}_{4}$ was added to make the protein solution up to 1 $\mathrm{M}\left(\mathrm{NH}_{4}\right)_{2} \mathrm{SO}_{4}$, equivalent to starting buffer A for $\mathrm{HIC}$ [ $50 \mathrm{mM}$ Bis-Tris and $\left.1 \mathrm{M}\left(\mathrm{NH}_{4}\right)_{2} \mathrm{SO}_{4}(\mathrm{pH} 7)\right]$. aSyn was eluted on a linear gradient against HIC buffer B [50 mM Bis-Tris ( $\mathrm{pH} 7)$ ] (Figure 1d.v, d.vi). Fractions containing aSyn after HIC were pooled and concentrated, and $500 \mu \mathrm{L}$ of aSyn was subsequently injected into a GF column and eluted isocratically (Figure 1d.vii). aSyn was $100 \%$ pure when analyzed by densitometry after IEX, HIC, and GF (Figure 2a and Table S1). Figure S2 and Table S2 show a second purification run for each purification method and the concentration of protein in each step during purification, showing that the methods are reproducible.

Densitometry analysis of the Coomassie blue-stained gel of the four aSyn samples after GF showed $100 \%$ pure monomeric aSyn in all samples (Figure 2a). However, analytical reversed phase chromatography ( $\mathrm{aRP}$ ) was also employed to determine the purity of each sample as it is a more sensitive method for detecting contaminants (Figure $2 \mathrm{~b}$ ). The samples were shown to be less pure after IEX by aRP compared to densitometry measurements (Figure S3a), and the sample purity ranged from $62.9 \%$ to $84.8 \%$ when analyzed by aRP but ranged between $49.7 \%$ and $100 \%$ when analyzed by densitometry (Table 1 and Table S1). aRP of aSyn purified by IEX, HIC, and GF compared 
Table 1. Purities of aSyn at Different Steps of the Purification Protocol Determined by Reverse Phase Chromatography

\begin{tabular}{|c|c|c|c|c|c|c|c|c|c|c|c|c|}
\hline & \multicolumn{3}{|c|}{ boiled } & \multicolumn{3}{|c|}{$\left(\mathrm{NH}_{4}\right)_{2} \mathrm{SO}_{4} \mathrm{ppt}$} & \multicolumn{3}{|c|}{ acid ppt } & \multicolumn{3}{|c|}{ periplasmic lysis } \\
\hline & $\begin{array}{c}\text { total } \\
\text { protein } \\
(\mathrm{mg} / \mathrm{mL})\end{array}$ & $\begin{array}{c}\% \\
\text { purity }\end{array}$ & $\begin{array}{c}\text { final aSyn } \\
\text { concentration } \\
(\mu \mathrm{M})\end{array}$ & $\begin{array}{c}\mathrm{tTotal} \\
\text { protein } \\
(\mathrm{mg} / \mathrm{mL})\end{array}$ & $\begin{array}{c}\% \\
\text { purity }\end{array}$ & $\begin{array}{c}\text { final aSyn } \\
\text { concentration } \\
(\mu \mathrm{M})\end{array}$ & $\begin{array}{c}\text { total } \\
\text { protein } \\
(\mathrm{mg} / \mathrm{mL})\end{array}$ & $\begin{array}{c}\% \\
\text { purity }\end{array}$ & $\begin{array}{c}\text { final aSyn } \\
\text { concentration } \\
(\mu \mathrm{M})\end{array}$ & $\begin{array}{c}\text { total } \\
\text { protein } \\
(\mathrm{mg} / \mathrm{mL})\end{array}$ & $\begin{array}{c}\% \\
\text { purity }\end{array}$ & $\begin{array}{c}\text { final aSyn } \\
\text { concentration } \\
(\mu \mathrm{M})\end{array}$ \\
\hline $\begin{array}{l}\text { post- } \\
\text { IEX }\end{array}$ & 1.038 & 69.3 & & 0.619 & 84.8 & & 1.59 & 62.9 & & 0.458 & 82.5 & \\
\hline $\begin{array}{l}\text { post- } \\
\text { HIC }\end{array}$ & - & - & & - & - & & - & - & & 0.254 & 86.7 & \\
\hline GF & 0.219 & 86 & 36.9 & 0.267 & 81 & 44.7 & 0.257 & 89.9 & 42.9 & 0.292 & 95 & 48.7 \\
\hline
\end{tabular}
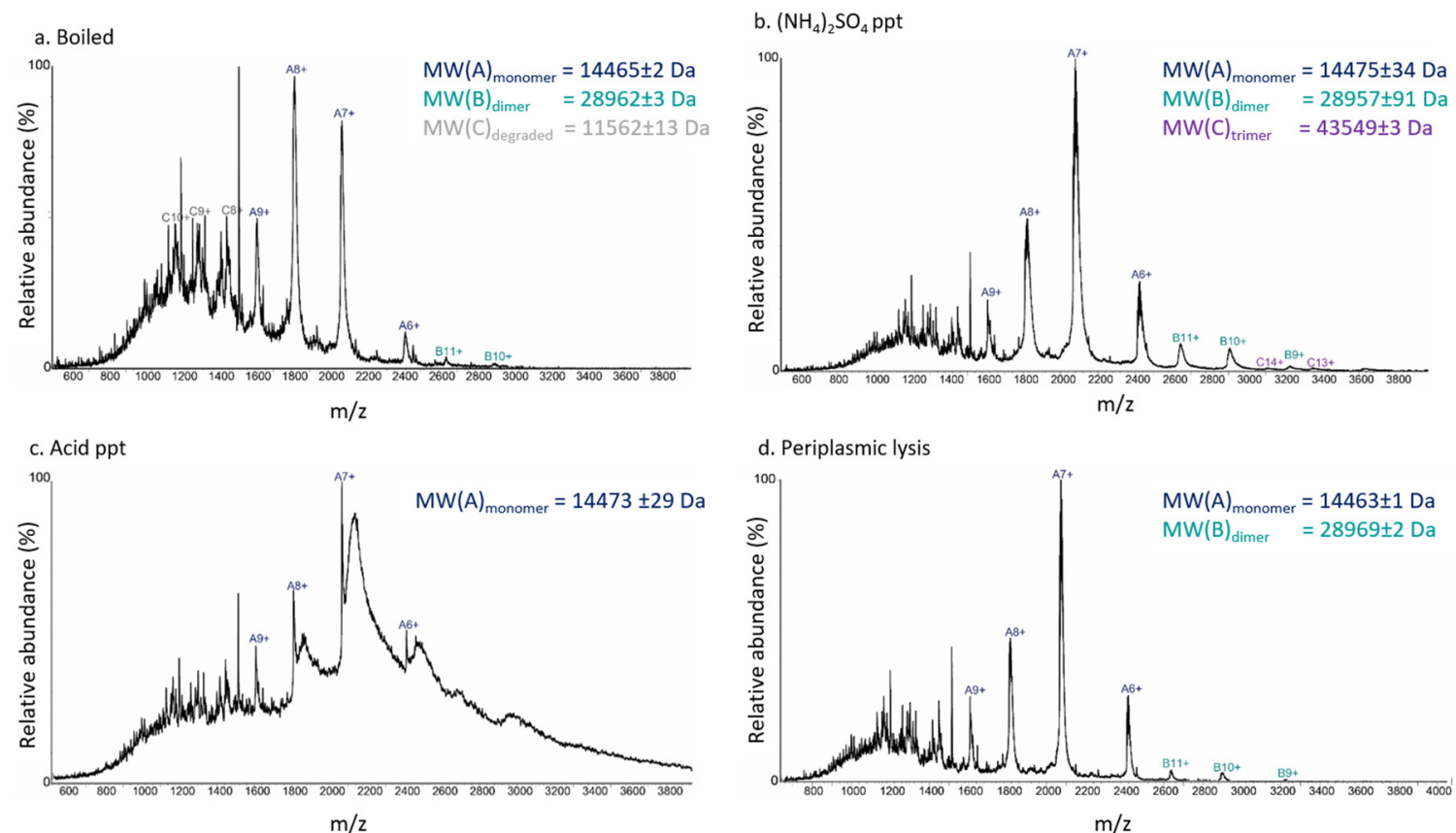

Figure 3. Native MS data of aSyn after gel filtration show that acid precipitation yields the highest percentage of monomeric aSyn. aSyn in $200 \mathrm{mM}$ $\mathrm{NH}_{4} \mathrm{CH}_{3} \mathrm{CO}_{2}$ was analyzed by native MS. aSyn isolated by (a) boiling was found as a monomer (A) in charge states 9+ to $6+$ where the MW is highlighted in blue, as a dimer (B) highlighted in teal in charge states $10+$ and $11+$, and as a potentially degraded product (C) highlighted in gray at charge states $10+$ to $8+$ with a $\mathrm{MW}$ of $\sim 11562 \mathrm{Da}$. aSyn isolated with (b) $\left(\mathrm{NH}_{4}\right)_{2} \mathrm{SO}_{4}$ ppt was identified as monomeric (A), dimeric (B), and trimeric (C) ( 43549 Da) protein forms; the trimer charge states $14+, 13+$, and MW are colored purple. The aSyn sample isolated with (c) acid ppt was found in only a monomeric state (A), while aSyn isolated by (d) periplasmic lysis was found to be in monomeric (A) and dimeric (B) forms.

Table 2. Molecular Weights and Relative Percentages of aSyn Structures in Each Sample as Determined by Native Nano-ESI-MS

\begin{tabular}{|c|c|c|c|c|c|c|c|c|}
\hline & \multicolumn{2}{|c|}{ boiled } & \multicolumn{2}{|c|}{$\left(\mathrm{NH}_{4}\right)_{2} \mathrm{SO}_{4} \mathrm{ppt}$} & \multicolumn{2}{|c|}{ acid ppt } & \multicolumn{2}{|c|}{ periplasmic lysis } \\
\hline & MW & $\%$ & MW & $\%$ & MW & $\%$ & MW & $\%$ \\
\hline degraded & $11562 \pm 13$ & 36.6 & - & - & - & - & - & - \\
\hline monomer & $14465.4 \pm 2.2$ & 60.2 & $14475 \pm 34$ & 90.3 & $14473 \pm 29$ & 100 & $14463 \pm 1$ & 96.5 \\
\hline dimer & $28962.5 \pm 3.0$ & 3.2 & $28957 \pm 91$ & 8.5 & - & - & $28969 \pm 2$ & 3.5 \\
\hline trimer & - & - & $43549 \pm 3$ & 1.2 & - & - & - & - \\
\hline
\end{tabular}

to only IEX and GF led to an increase in purity from $63.5 \%$ to $95 \%$ (Figure $\mathrm{S} 3 \mathrm{~b}$ ). After GF, the aSyn purity was determined to be $86 \%$ for the boiled sample, $81 \%$ for the $\left(\mathrm{NH}_{4}\right)_{2} \mathrm{SO}_{4} \mathrm{ppt}$ sample, $89.9 \%$ for the acid ppt sample, and $95 \%$ for periplasmic lysis of aSyn by aRP (Figure $2 \mathrm{~b}$ and Table 1 ).

Analysis of aSyn Samples by Native Mass Spectrometry Shows That the Acid Precipitation Protocol Yields Highly Monomeric aSyn. Highly pure monomeric aSyn is needed for the majority of assays performed. Although the SDS-PAGE gel stained with Coomassie blue and aRP methods show monomeric aSyn, the SDS used in the PAGE and the organic solvents used in $\mathrm{aRP}$ are denaturing and may give a false impression of the level of monomeric protein present. Instead, we employed nondenaturing nano-electrospray ionization mass spectrometry (native MS). The technique permits the study of protein structure at physiological $\mathrm{pH}$ and the identification of aSyn multimers and degradation products without the need to use cross-linkers that might alter structure or induce artifacts. ${ }^{18}$ In the boiled sample, aSyn was found in both monomer and dimer form, but also as a degraded product of $11562 \pm 3 \mathrm{Da}$ comprising $36.6 \%$ of the sample (Figure $3 a$ and Table 2). A degraded product of 12172 Da was also identified by Giehm et al., after boiling of aSyn. ${ }^{6}$ The percentage of aSyn products was calculated by the relative intensity of the $\mathrm{m} / z$ peaks in each charge state (Table S3). As the degraded product was not detected by SDS-PAGE or aRP, it may have been induced 


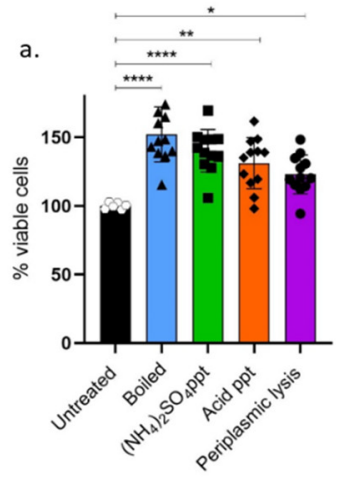

b.i
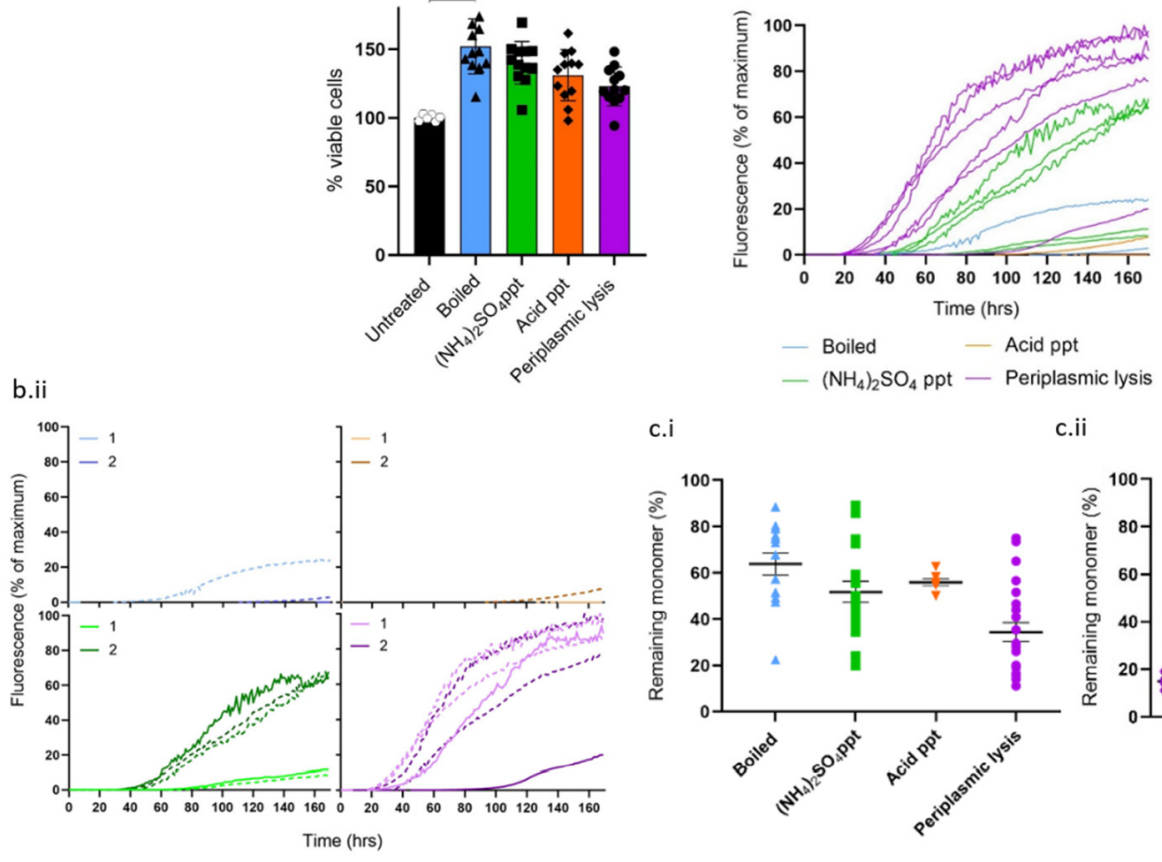

c.ii

d.
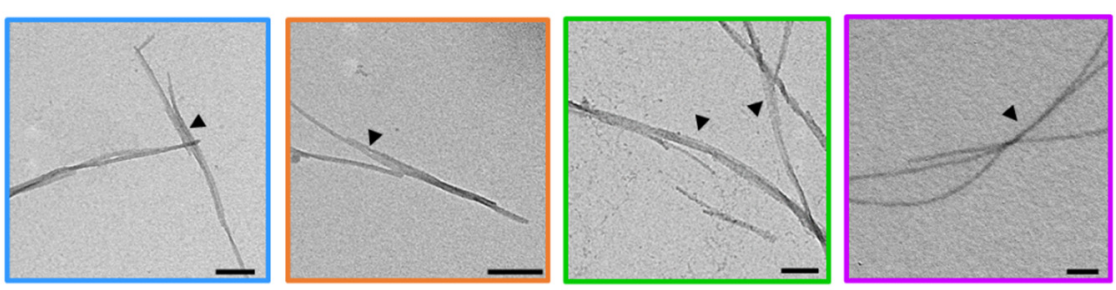

Figure 4. Incubation with aSyn purified by periplasmic lysis leads to the smallest increase in cell vitality and yields the most aggregation prone aSyn sample. (a) aSyn $(2 \mu \mathrm{M})$ from each purification protocol was incubated with SH-SY5Y cells for $24 \mathrm{~h}$, and a cell vitality test was performed using an MTT assay. Overall, cells were more viable after treatment with aSyn. Significant differences in cell vitality were found between all purification methods. Absorption data were normalized to untreated cells in each experiment. Individual data points are shown from four wells for each condition for three experiments. Error bars represent the standard error of the mean (SEM). Statistics were determined using a one-way analysis of variance with Holm-Sidak's tests $(* * * * p<0.0001 ; * * p=0.0027$; $*=0.0348)$. (b.i) ThT-based aggregation assays show aSyn samples isolated by periplasmic lysis (purple; $n=6$ ) and $\left(\mathrm{NH}_{4}\right)_{2} \mathrm{SO}_{4} \mathrm{ppt}$ (green; $\left.n=5\right)$ aggregate at a faster rate and to a greater extent than aSyn samples isolated by boiling (blue; $\left.n=4\right)$ or acid ppt (orange; $n=3$ ). Each sample $(n)$ represents the average of four well replicates. Twenty micromolar aSyn in $20 \mathrm{mM}$ Tris and $100 \mathrm{mM} \mathrm{KCl}(\mathrm{pH}$ 7.2) was incubated with $20 \mu \mathrm{M}$ ThT in a half-area 96-well plate with double orbital agitation at $300 \mathrm{rpm}$ for $5 \mathrm{~min}$ before each read every hour for $170 \mathrm{~h}$. (b.ii) aSyn ThT data are presented to show purification batches (lighter color, batch 1; darker color, batch 2) and samples from experiment 1 (solid lines) and experiment 2 (dashed lines) (individual well data are shown in Figure S5). (c.i) The percentage of the remaining monomer concentration in each well after the ThT-based assay was determined by performing SEC-HPLC and calculating the area under the curve compared to the area under the curve of the $20 \mu \mathrm{M}$ of starting monomeric sample. Error bars represent the SEM from wells: $n=14$ for boiled, $n=19$ for $\left(\mathrm{NH}_{4}\right)_{2} \mathrm{SO} \mathrm{P}_{4} \mathrm{ppt}, n=7$ for acid ppt, and $n=22$ for periplasmic lysis. (c.ii) Remaining monomer data are also displayed for each experiment and purification batch to display the variability between experiments and purification batches. (d) TEM images of aSyn samples after ThT assays showed fibrils with a straight morphology and lateral binding between fibril bundles (black arrows). The scale bar is $200 \mathrm{~nm}$. aSyn samples were taken directly from the ThT wells. Boiled (blue), $\left(\mathrm{NH}_{4}\right)_{2} \mathrm{SO}_{4}$ ppt (green), and periplasmic lysis (purple) samples were diluted 1:4, and the acid ppt (orange) sample was used neat when incubated on grids before being stained and imaged.

during electrospray ionization. aSyn samples precipitated in $\left(\mathrm{NH}_{4}\right)_{2} \mathrm{SO}_{4}$ contained monomer (90.3\%), dimer (8.5\%), and trimer (1.2\%) (Figure $3 \mathrm{~b}$ and Table 2 ), while aSyn isolated by acid ppt contained only monomeric aSyn (Figure $3 \mathrm{c}$ and Table 2). aSyn isolated by periplasmic lysis was highly monomeric (96.5\%) with a small percentage of dimers (3.5\%) (Figure 3d and Table 2). The monomer was disordered in all samples, as expected, and the dimer and trimers were possibly linked by noncovalent bonds that remain formed during nondenaturing electrospray ionization.
The Vitality of Cells Treated with aSyn and Aggregation Propensity of aSyn Differed among the Various Purification Protocols. To determine whether the difference in purity or the percentage of monomeric aSyn affects the cell response or the aSyn aggregation rate, we performed cell vitality and kinetic aggregation assays. SH-SY5Y cells, undifferentiated neuroblastoma cells, were incubated with $0.5-2 \mu \mathrm{M}$ aSyn from each purification method for $24 \mathrm{~h}$. Metabolic stress was then measured using an MTT assay that determines the metabolic ability of the cells to convert 3-(4,5-dimethylthiazol-2-yl)-2,5diphenyltetrazolium bromide (MTT) into an insoluble 
compound, formazan, which absorbs at $590 \mathrm{~nm}$. Under the experimental conditions, cells treated with aSyn at all concentrations had increased viability compared to that of the untreated cells (Figure 4a and Figure S4). There were significant differences in metabolic stress depending on the purification method used, with aSyn isolated by boiling and $\left(\mathrm{NH}_{4}\right)_{2} \mathrm{SO}_{4} \mathrm{ppt}$ leading to the most significant increases in cell vitality (152.2 \pm $20 \%$ and $140.2 \pm 15.5 \%$ increases, respectively) compared to untreated cells, while aSyn isolated by periplasmic lysis (123 \pm $14.2 \%)$ and acid ppt $(131.1 \pm 18.6 \%)$ led to less significant differences in cell vitality compared to untreated cells. The cell vitality data were also analyzed between batches 1 and 2 of purification, but no significant differences in cell vitality were determined between batches of purified aSyn (Figure S4).

The aggregation propensity of aSyn isolated by each method was then analyzed using the ThT molecule that fluoresces when bound to fibrillar forms of aSyn, providing a kinetic readout. ${ }^{19}$ Twenty micromolar aSyn was incubated with $20 \mu \mathrm{M}$ ThT in 20 $\mathrm{mM}$ Tris and $100 \mathrm{mM} \mathrm{KCl}$ ( $\mathrm{pH}$ 7.2) for 1 week. The kinetic aggregation curves show that the aSyn isolated by periplasmic lysis was the most prone to aggregation, followed by aSyn isolated by $\left(\mathrm{NH}_{4}\right)_{2} \mathrm{SO}_{4}$ ppt (Figure 4b.i). aSyn isolated by boiling and acid ppt appeared to be the least prone to aggregation under the conditions tested, barely aggregating (Figure 4b.i and Figure S5). There was great variation in the rate of aggregation observed between aSyn isolated from the different purification methods (Figure 4b.i and Table S4). We then further investigated variation between experiments and purification batches. The largest variation in aggregation propensity occurred between experiments rather than between purification batches, most clearly observed in the $\left(\mathrm{NH}_{4}\right)_{2} \mathrm{SO}_{4}$ ppt aSyn sample [Figure 4b.ii (green) and Figure S5b.iii]. However, there was also variation within the batches and between experiments, particularly in the periplasmic lysis aSyn sample [Figure 4b.ii (dark purple), Figure S5b.iv, and Tables S5 and S6]. The lag time $\left(t_{\text {lag }}\right)$ and the time to reach $50 \%$ of the maximum fluorescence $\left(t_{50}\right)$ were also calculated for each kinetic trace shown in Figure $4 \mathrm{~b} . \mathrm{i}$, and $t_{\mathrm{lag}}$ showed in general greater variation than $t_{50}$, suggesting nucleation rates are more affected by the purification method used compared to elongation rates between experiments and batches (Tables S4-S6).

Due to the high variation observed within ThT-based kinetic assays presented, and with the knowledge that ThT also has varying fluorescence intensities when bound to different fibril polymorphs, ${ }^{19,20}$ we performed analytical size exclusion chromatography with HPLC (SEC-HPLC) to quantify the amount of remaining aSyn monomer and therefore determine whether the extent of aggregation observed by the ThT-based assay was reflective. We observe that the quantity of the remaining aSyn monomer does not fully reflect the extent of ThT fluorescence observed, particularly for the acid ppt sample, but does reflect the overall aggregation trends observed in the ThT-based assays (Figure 4c.i). The remaining monomer data were further analyzed for variation between purification batch and experiment and more clearly show, compared to the ThTbased assays, that there was a greater variation in the percentage of the remaining monomer in the second experiment compared to the first (Figure 4c.ii). There appeared to be no clear correlation between aggregation propensity and either the purity or the proportion of the monomer in the starting sample.

We subsequently examined the morphology of aSyn samples after aggregation assays using TEM to determine whether fibrils had formed and whether their morphology differed. TEM showed that fibrils are present in all samples, but fibrils are harder to find in the sample purified by acid ppt, indicating fewer fibrils are present. All fibrils have a straight morphology (Figure $4 \mathrm{~d}$ and Figure S6), as shown previously for aSyn aggregated in the presence of salt. ${ }^{21}$ The fibril bundles also showed lateral binding (Figure $4 \mathrm{~d}$, shown by the arrows).

\section{DISCUSSION}

Many different protocols are currently used for the purification of aSyn, yet few investigations have been performed with the aSyn product that is present at the end of these purification methods and whether there are subsequent differences in downstream assays. Here, we compared four commonly applied protocols: boiling, acid ppt, $\left(\mathrm{NH}_{4}\right)_{2} \mathrm{SO}_{4} \mathrm{ppt}$, and periplasmic lysis. Isolation of aSyn by acid ppt and periplasmic lysis yielded the highest percentage of monomeric protein, at $100 \%$ and $96.5 \%$, respectively, and $89.9 \%$ and $95 \%$ purity, respectively. We observed variability in the resulting aggregation rate and cell vitality assays with aSyn from different purification protocols. We conclude that the purification method used affects the resulting recombinant aSyn, yet the precise origin of this variability is not currently clear.

The vitality of undifferentiated SH-SY5Y cells, using an MTT assay, was tested upon exposure to the four resulting aSyn samples, and the vitality of SH-SY5Y cells increased the most by the addition of aSyn isolated by boiling and $\left(\mathrm{NH}_{4}\right)_{2} \mathrm{SO}_{4}$ ppt. It has been previously observed that high levels of aSyn can lead to the proliferation of undifferentiated SH-SY5Y cells, aiding the tumorlike characteristics of the cell line and likely an increase in vitality. ${ }^{22}$ Similarly, differentiated SH-SY5Y cells treated with aSyn also had increased cell vitality and activation of the Akt pathway, a pro-survival pathway. ${ }^{23}$ Differences in cell vitality after treatment with aSyn from different purification methods indicate that the combination of lower purity and the presence of aSyn multimers and/or degradation products can affect the cell response to aSyn addition.

However, the rate of aggregation of aSyn was not greatly influenced by the purity or presence of multimers or degradation products. The difference in aSyn aggregation propensity between the aSyn samples isolated by the different purification methods was slightly surprising. As different ThT intensities can be observed from different fibril polymorphs, ${ }^{20}$ we also assessed the extent of aggregation by measuring the amount of remaining monomer after the assays and showed that the extent of aggregation by ThT-based assays was reflected in the amount of the remaining aSyn monomer after the assays. Both the remaining monomer percentage and ThT-based fluorescence assays had displayed greater variation between experiments rather than between purification batches, which could be the result of freezing and thawing of the samples, which can introduce multimers or degrade the sample. ${ }^{24}$

There was no clear correlation between the percentage of initial monomer or the presence of multimers and degradation products and the aggregation rate or remaining monomer percentage. This may instead indicate that the method of aSyn isolation can impact the dynamic ensemble of monomer conformations and the proportion of different conformations present, particularly for methods that disrupt intramolecular bonding such as boiling, acid precipitation, and $\left(\mathrm{NH}_{4}\right)_{2} \mathrm{SO}_{4}$ precipitation. We observe that the nucleation rate was more varied than the elongation rate among aSyn samples isolated by the four different protocols, potentially indicating that the purification method used affects the monomer state. Ion 
mobility mass spectrometry (IM-MS) has shown the presence of at least four main aSyn conformers, the distribution of which changes upon addition of ions or small molecules. ${ }^{25,26}$ This technique may be used to identify differences in the monomer conformer distribution of aSyn purified by the four protocols; however, these are potentially very small differences that may not be detected or may not be present in the gas phase during IM-MS.

We performed an array of characterization experiments on the four resulting recombinant aSyn products. We have highlighted that the method of purification used to produce recombinant aSyn can significantly affect the purity, the percentage of the monomer, and subsequent downstream assays, yet we have not been able to pinpoint the cause of variation in the rate of aggregation of aSyn when using the boiling, acid ppt, $\left(\mathrm{NH}_{4}\right)_{2} \mathrm{SO}_{4} \mathrm{ppt}$, or periplasmic lysis protocol. Further work is needed to determine if the purification protocol chosen influences aSyn monomer conformations and the dynamic ensemble of conformations. This may have subsequent effects on downstream assays, even potentially leading to changes in the subsequent oligomer and fibril polymorphs formed and their toxicity. ${ }^{24}$ It is thus important at the very least to characterize the aSyn sample fully in a bid to increase reproducibility and validity and to understand more clearly the downstream data acquired.

\section{ASSOCIATED CONTENT}

\section{SI Supporting Information}

The Supporting Information is available free of charge at https://pubs.acs.org/doi/10.1021/acs.biochem.0c00725.

Figures S1-S7 and Tables S1-S6 (PDF)

\section{Accession Codes}

P37840 (SYUA_HUMAN).

\section{AUTHOR INFORMATION}

\section{Corresponding Authors}

Amberley D. Stephens - Chemical Engineering and Biotechnology, University of Cambridge, Cambridge CB3 OAS, U.K.; (1) orcid.org/0000-0002-7303-6392; Email: asds2@ cam.ac.uk

Gabriele S. Kaminski Schierle - Chemical Engineering and Biotechnology, University of Cambridge, Cambridge CB3 OAS, U.K.; ○ orcid.org/0000-0002-1843-2202; Email: gsk20@ cam.ac.uk

\section{Authors}

Dijana Matak-Vinkovic - Department of Chemistry, University of Cambridge, Cambridge CB2 1EW, U.K.

Ana Fernandez-Villegas - Chemical Engineering and Biotechnology, University of Cambridge, Cambridge CB3 OAS, U.K.

Complete contact information is available at: https://pubs.acs.org/10.1021/acs.biochem.0c00725

\section{Author Contributions}

A.D.S. designed experiments and performed purification, kinetic assays, and TEM. D.M.-V. performed native MS. A.F.-V. performed cell culture and MTT assays. A.D.S. made the figures. A.D.S. and G.S.K.S. wrote the manuscript. All authors have given final approval of the manuscript.

\section{Funding}

G.S.K.S. acknowledges funding from the Wellcome Trust $(065807 / \mathrm{Z} / 01 / \mathrm{Z}$ and $203249 / \mathrm{Z} / 16 / \mathrm{Z})$, the UK Medical
Research Council (MRC) (MR/K02292X/1), Alzheimer Research UK (ARUK) (ARUK-PG013-14), the Michael J. Fox Foundation (16238), and Infinitus China Ltd.

\section{Notes}

The authors declare no competing financial interest.

Raw data are available at the University of Cambridge Data Repository (10.17863/CAM.58565).

\section{ACKNOWLEDGMENTS}

The authors thank Dr. Penny Hamyln for helpful discussions on chromatography protocols. The authors thank Lyn Carter and Filomena Gallo of the Cambridge Advanced Imaging Centre for their support and assistance in this work.

\section{REFERENCES}

(1) Duong-Ly, K. C., and Gabelli, S. B. (2014) Salting out of proteins using ammonium sulfate precipitation. In Methods in Enzymology, pp 85-94, Academic Press Inc.

(2) Huang, C., Ren, G., Zhou, H., and Wang, C. (2005) A new method for purification of recombinant human alpha-synuclein in Escherichia coli. Protein Expression Purif. 42, 173-177.

(3) Frimpong, A. K., Abzalimov, R. R., Uversky, V. N., and Kaltashov, I. A. (2010) Characterizationof intrinsically disordered proteins with electrospray inization mass spectrometry: Conformationl heterogeneity of $\alpha$-synuciein. Proteins: Struct., Funct., Genet. 78, 714-722.

(4) Gallat, F., Laganowsky, A., Wood, K., Gabel, F., van Eijck, L., Wuttke, J., Moulin, M., Hä rtlein, M., Eisenberg, D., Colletier, J., Zaccai, G., and Weik, M. (2012) Dynamical Coupling of Intrinsically Disordered Proteins and Their Hydration Water: Comparison with Folded Soluble and Membrane Proteins. Biophys. J. 103, 129-136.

(5) Ariesandi, W., Chang, C. F., Chen, T. E., and Chen, Y. R. (2013) Temperature-Dependent Structural Changes of Parkinson's AlphaSynuclein Reveal the Role of Pre-Existing Oligomers in AlphaSynuclein Fibrillization. PLoS One 8, No. e53487.

(6) Giehm, L., Lorenzen, N., and Otzen, D. E. (2011) Assays for $\alpha$ synuclein aggregation. in Methods, pp 295-305, Academic Press, Inc.

(7) Coelho-Cerqueira, E., Carmo-Gonçalves, P., Sá Pinheiro, A., Cortines, J., and Follmer, C. (2013) $\alpha$-Synuclein as an intrinsically disordered monomer - Fact or artefact? FEBS J. 280, 4915-4927.

(8) Uversky, V. N., Li, J., and Fink, A. L. (2001) Evidence for a Partially Folded Intermediate in $\alpha$-Synuclein Fibril Formation. J. Biol. Chem. 276, 10737-10744.

(9) McClendon, S., Rospigliosi, C. C., and Eliezer, D. (2009) Charge neutralization and collapse of the $\mathrm{C}$-terminal tail of alpha-synuclein at low pH. Protein Sci. 18, 1531-1540.

(10) Stephens, A. D., Nespovitaya, N., Zacharopoulou, M., Kaminski, C. F., Phillips, J. J., and Kaminski Schierle, G. S. (2018) Different Structural Conformers of Monomeric $\alpha$-Synuclein Identified after Lyophilizing and Freezing. Anal. Chem. 90, 6975-6983.

(11) Narhi, L., Wood, S. J., Steavenson, S., Jiang, Y., Wu, G. M., Anafi, D., Kaufman, S. A., Martin, F., Sitney, K., Denis, P., Louis, J.-C., Wypych, J., Biere, A. L., and Citron, M. (1999) Both familial Parkinson's disease mutations accelerate $\alpha$-synuclein aggregation. J. Biol. Chem. 274, 9843-9846.

(12) Conway, K. A., Harper, J. D., and Lansbury, P. T. (1998) Accelerated in vitro fibril formation by a mutant $\alpha$-synuclein linked to early-onset Parkinson disease. Nat. Med. 4, 1318-1320.

(13) Giasson, B. I., Uryu, K., Trojanowski, J. Q., and Lee, V. M. Y. (1999) Mutant and wild type human $\alpha$-synucleins assemble into elongated filaments with distinct morphologies in vitro. J. Biol. Chem. $274,7619-7622$.

(14) Uversky, V. N., Li, J., and Fink, A. L. (2001) Metal-triggered structural transformations, aggregation, and fibrillation of human $\alpha$ synuclein: A possible molecular link between parkinson's disease and heavy metal exposure. J. Biol. Chem. 276, 44284-44296.

(15) Schindelin, J., Arganda-Carreras, I., Frise, E., Kaynig, V., Longair, M., Pietzsch, T., Preibisch, S., Rueden, C., Saalfeld, S., Schmid, B., 
Tinevez, J. Y., White, D. J., Hartenstein, V., Eliceiri, K., Tomancak, P., and Cardona, A. (2012) Fiji: An open-source platform for biologicalimage analysis. Nat. Methods 9, 676-682.

(16) Heppel, L. A. (1969) The effect of osmotic shock on release of bacterial proteins and on active transport. J. Gen. Physiol. 54, 95-113.

(17) Campioni, S., Carret, G., Jordens, S., Nicoud, L., Mezzenga, R., and Riek, R. (2014) The Presence of an Air-Water Interface Affects Formation and Elongation of $\alpha$-Synuclein Fibrils. J. Am. Chem. Soc. 136, 2866-2875.

(18) Mitra, G. (2019) Application of native mass spectrometry in studying intrinsically disordered proteins: A special focus on neurodegenerative diseases. Biochim. Biophys. Acta, Proteins Proteomics 1867, 140260.

(19) Biancalana, M., and Koide, S. (2010) Molecular mechanism of Thioflavin-T binding to amyloid fibrils. Biochim. Biophys. Acta, Proteins Proteomics 1804, 1405-1412.

(20) Sidhu, A., Vaneyck, J., Blum, C., Segers-Nolten, I., and Subramaniam, V. (2018) Polymorph-specific distribution of binding sites determines thioflavin-T fluorescence intensity in $\alpha$-synuclein fibrils. Amyloid 25, 189-196.

(21) Bousset, L., Pieri, L., Ruiz-Arlandis, G., Gath, J., Jensen, P. H., Habenstein, B., Madiona, K., Olieric, V., Böckmann, A., Meier, B. H., and Melki, R. (2013) Structural and functional characterization of two alpha-synuclein strains. Nat. Commun. 4, 2575.

(22) Rodríguez-Losada, N., de la Rosa, J., Larriva, M., Wendelbo, R., Aguirre, J. A., Castresana, J. S., and Ballaz, S. J. (2020) Overexpression of alpha-synuclein promotes both cell proliferation and cell toxicity in human SH-SY5Y neuroblastoma cells. J. Adv. Res. 23, 37-45.

(23) Kim, J. Y., Jeon, B. S., Kim, H. J., and Ahn, T. B. (2013) Nanomolar concentration of alpha-synuclein enhances dopaminergic neuronal survival via Akt pathway. Neural Regener. Res. 8, 3269-3274.

(24) Polinski, N. K., Volpicelli-Daley, L. A., Sortwell, C. E., Luk, K. C., Cremades, N., Gottler, L. M., Froula, J., Duffy, M. F., Lee, V. M. Y., Martinez, T. N., and Dave, K. D. (2018) Best practices for generating and using alpha-synuclein pre-formed fibrils to model Parkinson's disease in rodents. J. Parkinson's Dis. 8, 303-322.

(25) Konijnenberg, A., Ranica, S., Narkiewicz, J., Legname, G., Grandori, R., Sobott, F., and Natalello, A. (2016) Opposite Structural Effects of Epigallocatechin-3-gallate and Dopamine Binding to $\alpha$ Synuclein. Anal. Chem. 88, 8468-8475.

(26) Stephens, A. D., Zacharopoulou, M., Moons, R., Fusco, G., Seetaloo, N., Chiki, A., Woodhams, P. J., Mela, I., Lashuel, H. A., Phillips, J. J., De Simone, A., Sobott, F., and Schierle, G. S. K. (2020) Extent of N-terminus exposure of monomeric alpha-synuclein determines its aggregation propensity. Nat. Commun. 11, 2820. 